\title{
Antihypertensive Effects of Total Gastrectomy on Spontaneous Hypertensive Rats
}

\author{
Naoki Hashimoto \\ Department of Emergency Medicine, Sanda City Hospital, Sanda, Japan \\ Email: gojigen000@gmail.com
}

How to cite this paper: Hashimoto, N. (2020) Antihypertensive Effects of Total Gastrectomy on Spontaneous Hypertensive Rats. Open Access Library Journal, 7: e5994. https://doi.org/10.4236/oalib.1105994

Received: December 11, 2019

Accepted: January 10, 2020

Published: January 13, 2020

Copyright $\odot 2020$ by author(s) and Open Access Library Inc.

This work is licensed under the Creative Commons Attribution International License (CC BY 4.0).

http://creativecommons.org/licenses/by/4.0/

\begin{abstract}
Backgrounds: Total gastrectomy (TG) played an important role in DM improvement after curative surgery for gastric cancer (W J Surgery 2014, 58, 451-0458). The aim of the present study was to establish the effects of TG on weight loss and cardiovascular (CV) parameters in spontaneous hypertensive rats (SHR). Methods: Male SHR rats were used in the present studies. Rats were randomly assigned to either total gastrectomy (TG) or sham (Control): We measured blood pressure, heart rate, body weight and renin-aldosterone from postoperative 1 week to 12 weeks once a week for TG and Control group. Results: Systolic and Diastolic Blood Pressure: TG is significantly lower compared to Control. Body Weight: TG is significantly lower compared to Control. But, there is no relationship between systolic blood pressure and body weight in TG. Renin and Aldosterone Renin and aldosterone in TG were significantly lower compared to those in control from 3 to 12 weeks. Conclusion: We demonstrated that TG lowered BP in SHR rats. Our data also showed that the beneficial effect of TG for BP was independent of weight loss in SHR rats. Our mechanistic evidence suggests that this CV benefit is likely to be the result of decreasing Renin-angiotensin-aldosterone system activities.
\end{abstract}

\section{Subject Areas \\ Emergency \& Critical Care}

\section{Keywords}

Spontaneous Hypertension Rat (SHR), Blood Pressure, Body Weight, Renin-Aldosterone, Sympathetic Nervous System (SNS), Total Gastrectomy (TG)

\section{Introduction}

Bariatric surgery is currently the most effective long-term treatment for obesity 
and results in high rates of remission for type 2 diabetics and hypertension [1] [2]. Furthermore, bariatric surgery produces a greater reduction in arterial blood pressure (AP) than medical management alone [3]. However, the mechanism by which this occurs remains elusive. Bariatric surgery has been adapted as a metabolic surgery that can not only reduce body weight but also improve symptoms of metabolic syndrome, such as hypertension, hyperlipidemia and Diabetic Mellitus (DM). Different types of bariatric surgery have been adapted, including R-Y gastric bypass, sleeve gastrectomy and adjustable gastric band.

The resolution of DM after bariatric surgery results from not only body weight loss itself but also changes in gut hormones, which are now being thoroughly investigated by many researchers [4] [5]. Kange et al. [6] suggested that forgut-bypass was the key factor that led to improvements in type 2 DM rather than body weight loss alone. The DM improvement rates in patients who underwent B-1, B-II and R-Y surgeries were $45.2 \%, 85.2 \%$ and $88.2 \%$, respectively. In that study, R-Y reconstruction was used when a patient received total gastrectomy, and B-1 and B-II reconstructions were applied in patients who received subtotal gastrectomies.

The aim of the present study was to establish the effects of total gastrectomy on weight loss and cardiovascular parameters in spontaneous hypertensive rats (SHR).

\section{Material and Methods}

Male SHR rats were used in the present studies. All procedures were conducted in accordance with the guidelines for the Care and Use of Laboratory Animals of the National Institutes of Health and were approved by the Ethical committee for Animal Experimentation of the University of Kindai (KAME-28-024).

Eight weeks male SHR rats were obtained from Kindai Life Science Institute, Osaka Japan. Rats were randomly assigned to either total gastrectomy (TG) or sham (Control).

\section{Surgical procedures}

The rats were permitted to acclimate for 4 weeks before surgery. Prior to surgery, the animals were fasted for 24 hours. Total gastrectomy and sham ope were performed under general anesthesia (somnolently $50 \mathrm{mg} / \mathrm{kg}$ body weight intraperitoneal injection) through an upper middle incision on 12 weeks.

Total gastrectomy $(\mathrm{TG})(\mathrm{n}=12)$ (Figure 1): The gastroesophageal junction was ligated and the distal esophagus was transected $2 \mathrm{~mm}$ above the ligature. Moreover, the gastroduodenal junction was also ligated, and the proximal duodenum was transected $3 \mathrm{~mm}$ distal to the pylorus. A total gastrectomy was performed with the removal of the entire stomach, and end-to-end anastomosis of the esophagus and duodenum.

Sham group (Control) $(n=8)$. Sham operation was performed.

We measured blood pressure, heart rate, body weight and renin-aldosterone from postoperative 1 week to 12 week once a week for TG and Control group. 

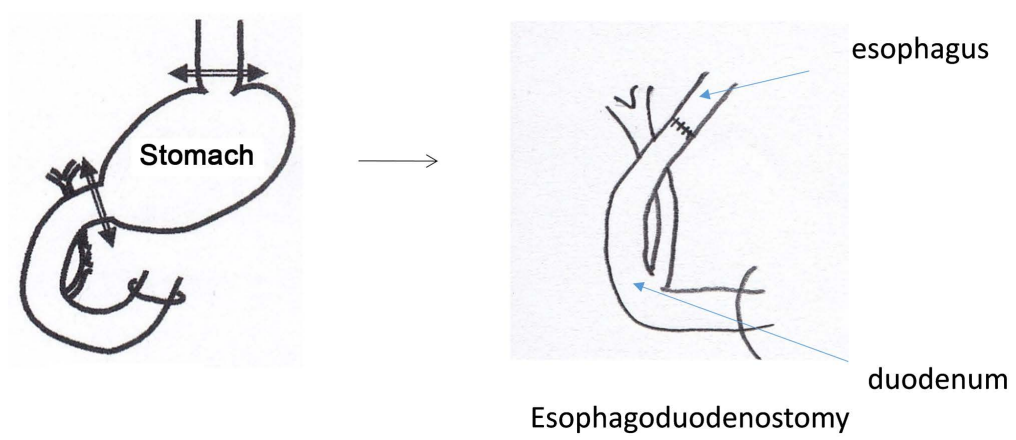

Figure 1. Total gastrectomy (Surgical procedure).

\section{Measurement of Blood pressure and Heart rate}

Blood pressure values as well as heart rate (HR) were recorded in conscious, resting animals by non-invasive tail-cuff plethymography (BP-98A-L Soltron, Tokyo, Japan).

After a 3 hour fast, the tail artery was dilated by placement of the animal into a thermostatically controlled plastic holder heated at $32^{\circ} \mathrm{C}$ for $20 \mathrm{~min}$. Tail pulse was detected by passage of the tail through a tail-cuff sensor attached to the amplifier.

All measurements were carried out between 10:00 and 12:00, and an average of a least three readings was taken for each animal after they became used to the environment.

Plasma level of Renin and aldosterone

Peripheral blood was drawn from tail vein for the measurement of renin and aldosterone at postoperative 1, 2, 3, 4, 7 and 10 weeks for TG and Control.

The determination of plasma aldosterone activity and renin activity was determined by radioimmunoassay.

Statistics

Comparisons between groups were made by the Mann-Whitney test. A difference between groups of $\mathrm{p}<0.05$ was considered statistically significant. Statistical analysis was performed using the Stat View 5.0-J program (Abacus Concepts, Berkeley, CA, USA.

\section{Results}

1) Changes of Systolic Blood Pressure in SHR rat (Figure 2).

TG was significantly lower compared to Control at postoperative 1 week. But TG gradually increased from postoperative 3 to 4 weeks. There is no difference between TG and Control from postoperative 2 to 4 weeks. But thereafter TG gradually decrease from postoperative 5 to 12 weeks. TG is significantly lower compared to Control from postoperative 5 to 12 weeks.

2) Changes of Diastolic Blood Pressure in SHR rat (Figure 3).

TG is significantly lower compared to Control from postoperative 1 to 12 weeks. Especially, TG in Blood pressure gradually decrease from postoperative 4 to 12 weeks. 


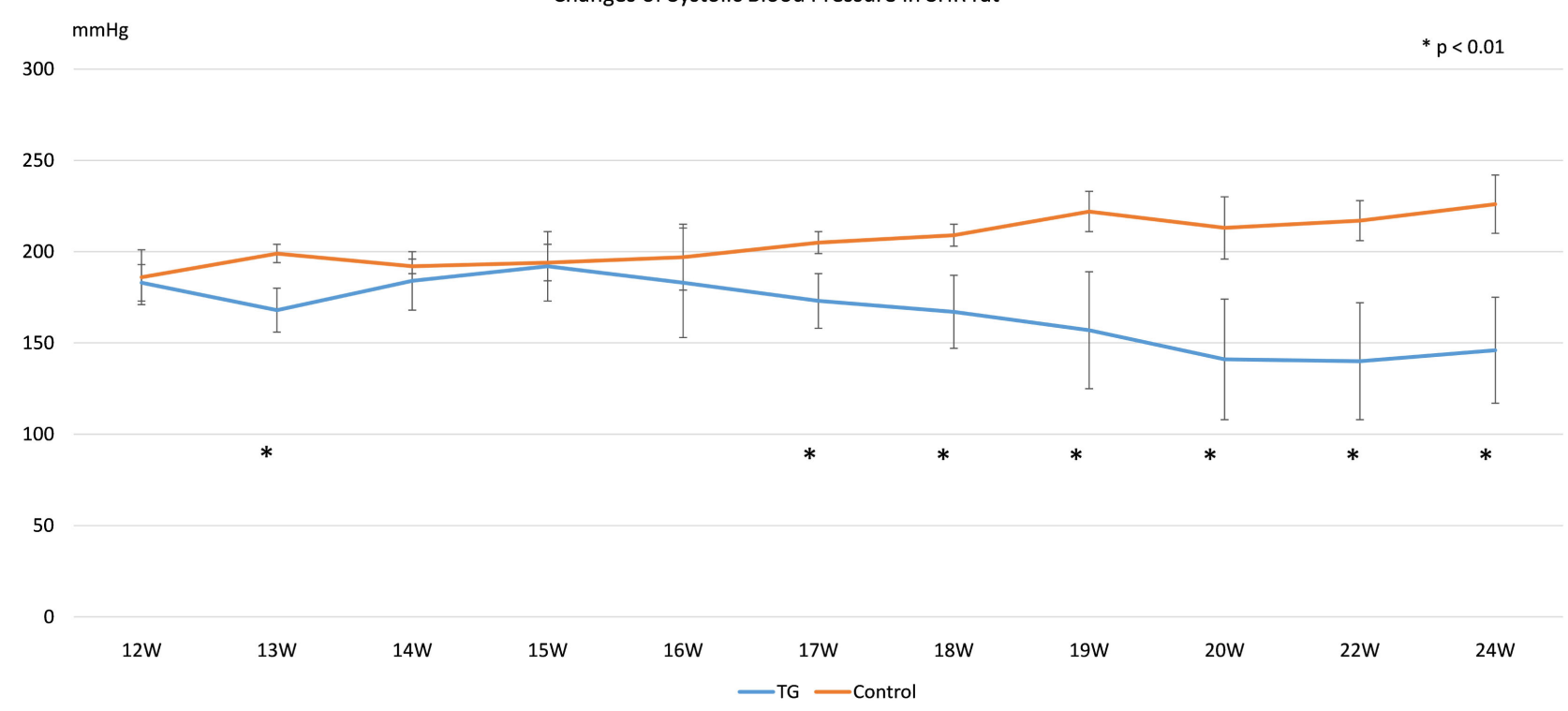

Figure 2. Changes of systolic blood pressure in SHR rat.

$\mathrm{mmHg} \quad$ Changes of Diastolic Blood Pressure in SHR rat

200

180

160

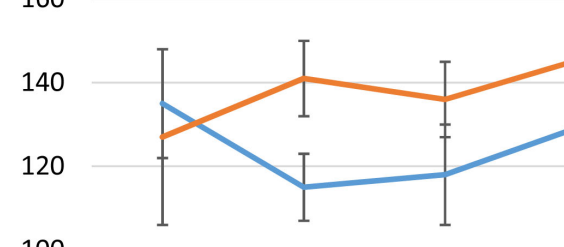

100

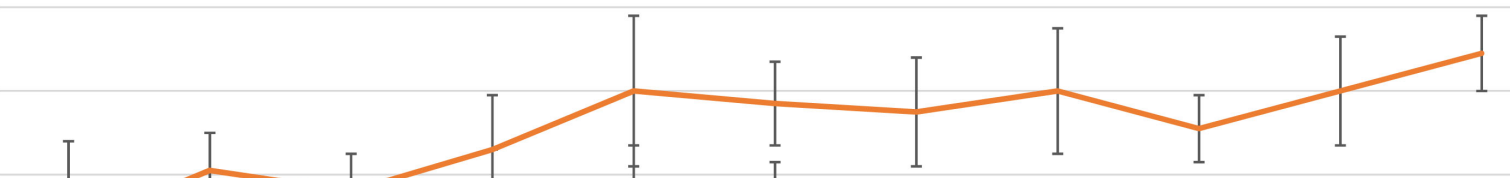

80

60

1

40

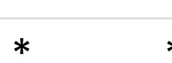

$*$

$* *$
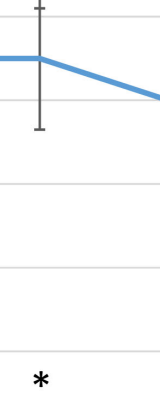

40

20

0

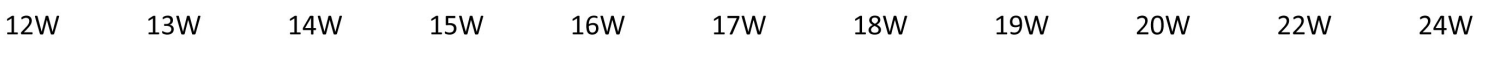

$\longrightarrow$ TG Control

Figure 3. Changes of diastolic blood pressure in SHR rat.

3) Changes of Body Weight in SHR rat (Figure 4).

TG is significant lower compared to Control from postoperative 1 to 12 weeks.

4) Relationship between Body Weight and Systolic Blood Pressure in SHR rat (Figure 5). 


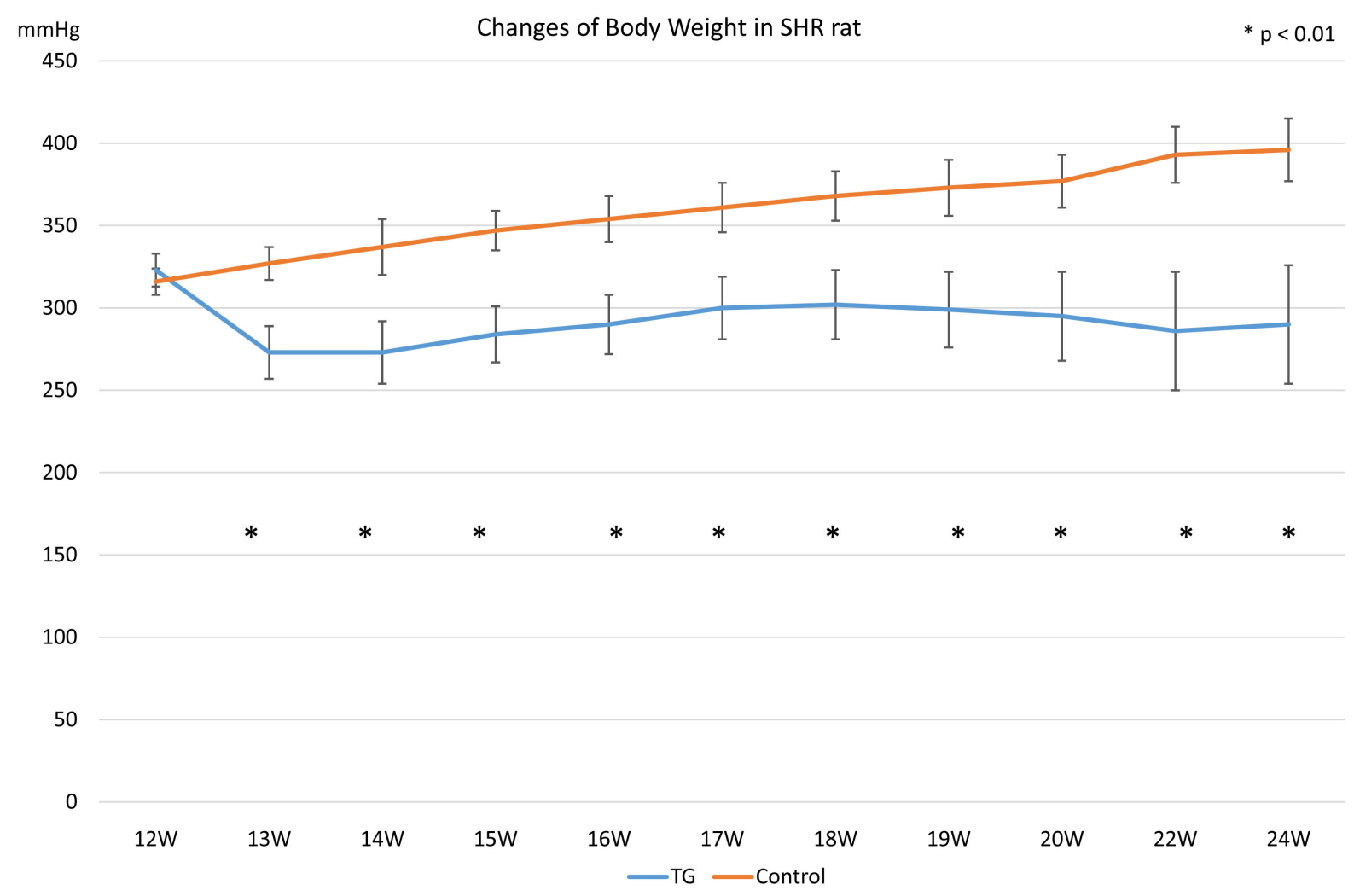

Figure 4. Changes of body weight in SHR rat.

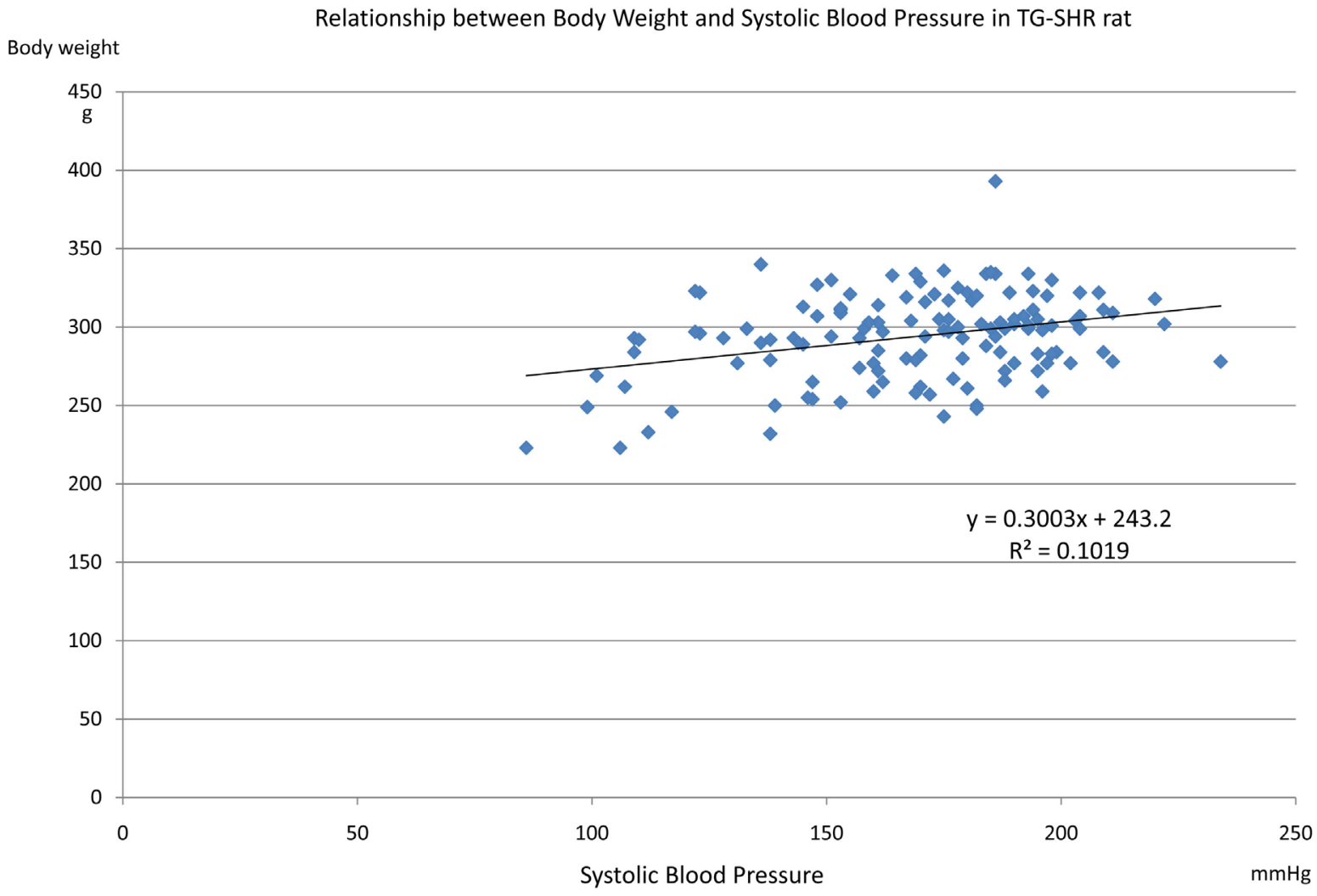

Figure 5. Relationship between body weight and systolic blood pressure in SHR rat. 
There is no correlation between body weight and Systolic Pressure in SHR rat. Therefore, we are not likely to believe that the decrease in blood pressure in TG is due to weight loss.

5) Plasma level of Renin and Aldosterone in SHR rat (Figure 6, Figure 7).

There is no difference between TG and Control for the postoperative 2 weeks.

Renin and aldosterone in TG were significantly lower compared to those in Control from postoperative 3 to 12 weeks.

\section{Discussion}

The present study provides evidence for the beneficial effect of TG on hypertension and cardio-metabolic risks. Our studies confirmed that gastrointestinal (GI) surgical intervention can significantly inhibit the overdrive of sympathetic nervous system (SNS) in genetic hypertensive rats [7], which results in reducing blood pressure and improvement of cardiovascular remodeling and dysfunction. Metabolic Surgery is currently the most effective treatment option for obesity and diabetes [8] [9]. In addition, metabolic surgery can effectively ameliorate hypertension over the long term [10] [11], however, the underlying mechanism remains elusive. Several studies suggest that the reduction in BP could be related to metabolic surgery-induced weight loss [12]. However, clinical trials and experimental studies indicated that a quick reduction in BP occurred before the

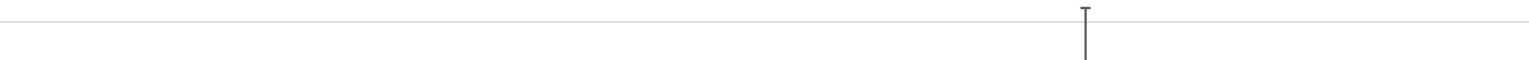

1

12

8

6

4

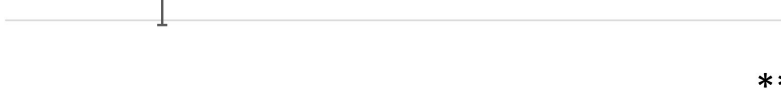

0

$13 \mathrm{~W}$

$14 \mathrm{~W}$

$15 \mathrm{~W}$

$16 \mathrm{~W}$

$22 \mathrm{~W}$ 


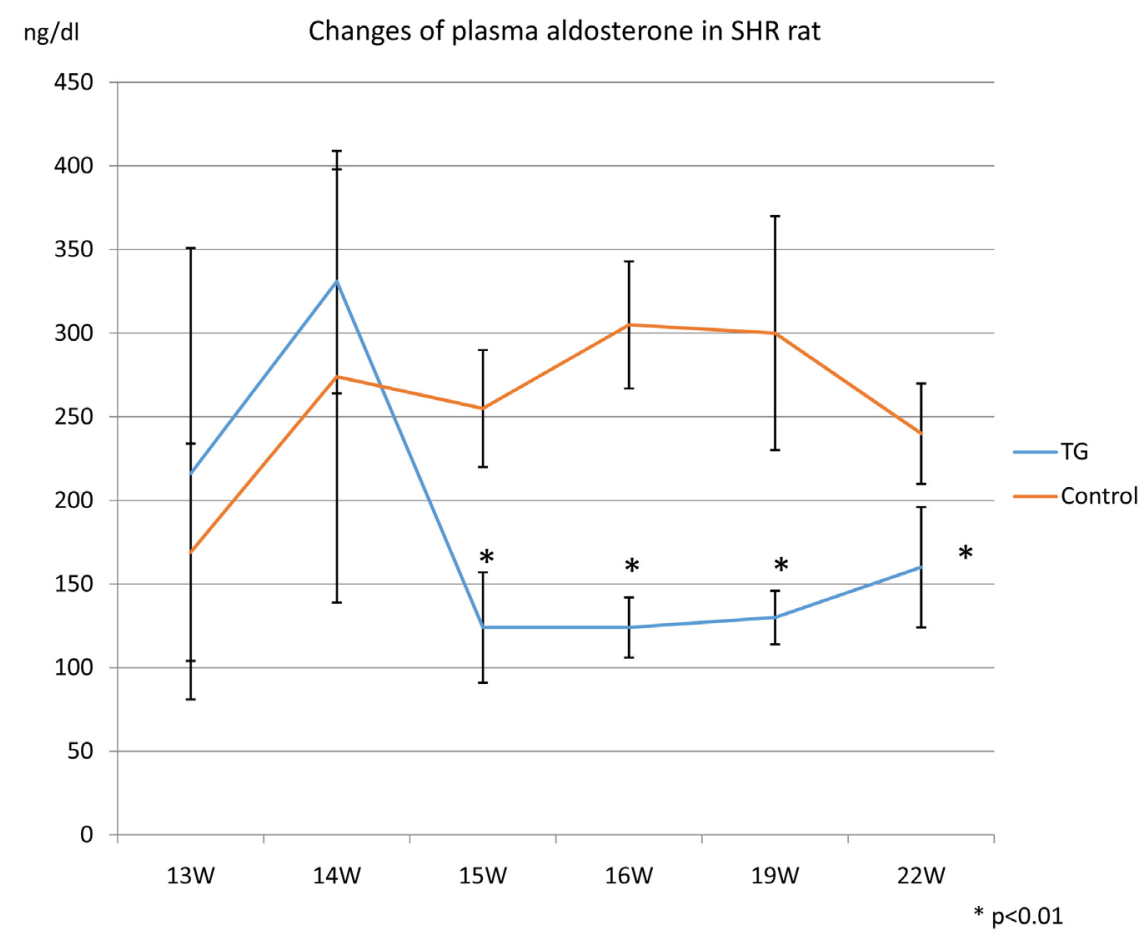

Figure 7. Plasma level of Aldosterone in SHR rat.

metabolic surgery medicated weight loss [13]. A meta analysis reported that there was resolution of hypertension in $61.7 \%$ of patients and resolution or improvement of hypertension in $78.5 \%$ of patients after metabolic surgery [14]. Antihypertensive therapy was reduced or discontinued in $70 \%$ of patients receiving metabolic surgery [15]. Our data also showed that the beneficial effect of TG for BP was independent of weight loss in surgically treated hypertensive rats. However, the mechanism underlying the metabolic surgery medicated antihypertensive effect remains unknown. Although the precise mechanism mediating hypertension remission after metabolic surgery remains poorly understood, it is apparent that rearrangement of GI anatomy can exert several discrete antihypertensive effects beyond those related to reduce sodium intake and body weight. Proposed multiple factors include the change in gut hormones, renin-angiotensin-aldosterone system and intestinal microbial flora [16] [17] [18]. Ruano et al. [19] proposed that the obese state is associated with raised levels of plasma renin activity, aldosterone, an angiotensin-converting enzyme leading to sodium retention and that after bariatric surgery these abnormal hormone levels tend to normalize. Our data also showed that TG decreases renin-aldosterone level compared to control. Sugerman [20] speculates a reduction in visceral fat and intraabdominal pressure after bariatric surgery might reduce renin-angiotensin-aldosterone activity, increases natriuresis and thus decrease arterial blood pressure. I agree with his opinion. Our findings provide insights into the mechanism of total gastrectomy in the regulation of BP. GI intervention may represent a promising intervention in resistant hypertension with metabolic disturbances. 


\section{Conclusion}

We demonstrated that TG lowers BP in SHR rats. Our mechanistic evidence suggests that this CV benefit is likely to be the result of decreasing Renin-aldosterone system activities. Our findings provide insights into the mechanism of metabolic surgery in the regulation of BP. GI intervention may represent a promising intervention in resistant hypertension with metabolic disturbances.

\section{Conflicts of Interest}

The author declares no conflicts of interest regarding the publication of this paper.

\section{References}

[1] Flores, L., Vidal, J., Canivell, S., et al. (2014) Hypertension Remission 1 Year after Bariatric Surgery: Predictive Factors. Surgery for Obesity and Related Diseases, 10, 661-665. https://doi.org/10.1016/j.soard.2013.11.010

[2] Tritsch, A.M., Bland, C.M., Hatzigeorgiou, C., et al. (2015) A Retrospective Review of the Medical Management of Hypertension and Diabetes Mellitus Following Sleeve Gastrectomy. Obesity Surgery, 25, 642-647.

https://doi.org/10.1007/s11695-014-1375-y

[3] Halperin, F., Ding, S.A., Simonson, D.C., et al. (2014) Roux-en-Y Gastric Bypass Surgery or Lifestyle with Intensive Medical Management in Patients with Type 2 Diabetes: Feasibility and 1-Year Results of a Randomized Clinical Trial. JAMA Surgery, 149, 716-726. https://doi.org/10.1001/jamasurg.2014.514

[4] Navarrete, A.S., Leyba, J., Navarrete, L.L.S., et al. (2012) Roux-en-Y Gastric Bypass for Treatment of Patients with DM Type 2 and BMI of $30-35 \mathrm{~kg} / \mathrm{m}^{2}$. Nutrición Hospitalaria, 27, 1160-1165.

[5] Gill, R.S., Birch, D.W., Shi, X., et al. (2010) Sleeve Gastrectomy and Type 2 Diabetes Mellitus: A Systemic Review. Surgery for Obesity and Related Diseases, 6, 707-713. https://doi.org/10.1016/j.soard.2010.07.011

[6] Kang, K.C., Shin, S.H., Lee, Y.J., et al. (2012) Influence of Gastrectomy for Stomach Cancer on Type 2 Diabetes Mellitus for Patients with a Body Mass Indexless than 30 $\mathrm{kg} / \mathrm{m}^{2}$. Journal of the Korean Surgical Society, 82, 347-355. https://doi.org/10.4174/jkss.2012.82.6.347

[7] Curry, T.B., Somaraju, M., Hines, C.N., et al. (2013) Sympathetic Support of Energy Expenditure and Sympathetic Nervous System Activity after Gastric Bypass Surgery. Obesity, 21, 480-485. https://doi.org/10.1002/oby.20106

[8] Cummings, D.E. (2012) Metabolic Surgery for Type 2 Diabetes. Nature Medicine, 18, 656. https://doi.org/10.1038/nm.2773

[9] Schauer, P.R., Kashyap, S.R., Wolski, K., et al. (2012) Bariatric Surgery versus Intensive Medical Therapy in Obese Patients with Diabetes. The New England Journal of Medicine, 366, 1567-1576. https://doi.org/10.1056/NEJMoa1200225

[10] Rodriguez, A., Becerril, S., Valenti, V., et al. (2012) Short-Term Effects of Sleeve Gastrectomy and Caloric Restriction on Blood Pressure in Diet-Induced Obese Rats. Obesity Surgery, 22, 1481-1490. https://doi.org/10.1007/s11695-012-0702-4

[11] Arteburn, D., Schauer, D.P., Wise, R.E., et al. (2009) Changes in Predicted 10-Year 
Cardiovascular Risk Following Laparoscopic Roux-en-Y Gastric Bypass Surgery. Obesity Surgery, 19, 184-189. https://doi.org/10.1007/s11695-008-9534-7

[12] Fernstrom, J.D., Courcoulas, A.P., Houck, P.R., et al. (2006) Long-Term Changes in Blood Pressure in Extremely Obese Patients Who Have Undergone Bariatric Surgery. The Archives of Surgery, 141, 276. https://doi.org/10.1001/archsurg.141.3.276

[13] Ahmed, A.R., Rickards, G., Conigio, D., et al. (2009) Laparoscopic Roux-en-Y Gastric Bypass and Its Early Effect on Blood Pressure. Obesity Surgery, 19, 845-849. https://doi.org/10.1007/s11695-008-9671-Z

[14] Buchwald, H., Avidor, Y., Braunwald, E., et al. (2004) Bariatric Surgery. JAMA, 292, 1724-1737. https://doi.org/10.1001/jama.292.14.1724

[15] Mingrone, G., Panunzi, S., De Gaetano, A., et al. (2012) Bariatric Surgery versus Conventional Medical Therapy for Type 2 Diabetes. The New England Journal of Medicine, 366, 1577-1585. https://doi.org/10.1056/NEJMoa1200111

[16] Wang, P., Yan, Z., Zhong, J., et al. (2012) Transient Receptor Potential Vanilloid 1 Activation Enhances Gut Glucagon-Like Peptide 1 Secretion and Improves Glucose Homeostasis. Diabetes, 61, 2155-2165. https://doi.org/10.2337/db11-1503

[17] Mayer, E.A. (2011) Gut Feelings: The Emerging Biology of Gut-Brain Communication. Nature Reviews Neuroscience, 12, 453-456. https://doi.org/10.1038/nrn3071

[18] Wang, B., Zhong, J., Lin, H., et al. (2013) Blood Pressure Lowering Effects of GLP-1 Receptor Agonists Exenatide and Liraglutide: A Meta-Analysis of Clinical Trials. Diabetes, Obesity and Metabolism, 15, 737-749. https://doi.org/10.1111/dom.12085

[19] Ruano, M., Silvestre, V., Gastro, R., et al. (2005) Morbid Obesity, Hypertensive Disease and the Renin-Angiotensin-Aldosterone Axis. Obesity Surgery, 15, 670-676. https://doi.org/10.1381/0960892053923734

[20] Sugerman, H.J. (1998) Increased Intra-Abdominal Pressure in Obesity. International Journal of Obesity and Related Metabolic Disorders, 22, 1138. https://doi.org/10.1038/sj.ijo.0800757 